\title{
Connectivity dynamics and cognitive variability during aging
}

Jauny, G. ${ }^{1}$, Eustache, F. ${ }^{1}, \&$ Hinault, T. ${ }^{* 1}$

${ }^{1}$ Normandie Univ, UNICAEN, PSL Université Paris, EPHE, INSERM, U1077, CHU de Caen, Centre Cyceron

*Corresponding author:

Thomas Hinault

INSERM-EPHE-UNICAEN U1077, 2 rue des Rochambelles, 14032 Caen, FRANCE.

Email: thomas.hinault@inserm.fr

Declarations of interest: none 


\begin{abstract}
Aging is accompanied by cognitive changes but strong variations across individuals exists. One of the ways to characterize this individual variability is to use techniques such as magnetoencephalography (MEG) to measure the dynamics of synchronization between brain regions and the variability of this connectivity over time. Indeed, few studies have focused on the fluctuations in the dynamics of brain networks over time and their evolution with age. We therefore used this method on the Cam-CAN database. We show here that with age an increase in the variability of brain synchronization, as well as a reversal of the direction of information transfer in the default mode network (DMN), in delta frequency band takes place. These changes in functional connectivity were associated with cognitive decline. These results suggest that advancing age is accompanied by a functional disorganization of dynamic networks with a loss of communication stability and a decrease in the information transmitted. This could be partly due to the loss of integrity of the network structure.
\end{abstract}

Keywords: Aging; MEG; Cognition; Cognitive variability; Connectivity 


\section{Introduction}

With an increasing number of people over 65, the world population is aging. Aging is associated with a reduced efficiency of cognitive functioning, that primarily affects memory and executive processes (e.g., Hedden \& Gabrieli, 2004). However, some individuals show a major decline while others maintain cognitive performance similar to young adults (e.g., Hultsch et al., 2008). Recent research aims to better understand these individual differences during aging. Such variability across individuals has been associated with concepts of maintenance and cognitive reserve (Cabeza et al., 2018; Stern et al., 2020). Maintenance corresponds to the preservation of similar cognitive and brain functioning to that of younger individuals with advancing age, while cognitive reserve corresponds to compensatory functional adjustments associated with the preservation of cognitive performance in the presence of structural changes. Cognitive reserve and maintenance can account for individual differences in aging- and pathology-related effects, and have been extensively investigated at both structural and functional levels (e.g., Stern et al., 2020). However, the contribution of the temporal dynamics of brain communications underlying cognitive reserve remains under-investigated. As changes in brain dynamics are expected to occur long before the disconnection associated with atrophy and brain lesions, this could yield highly sensitive elements on individual differences with age.

Neuroimaging research in healthy aging has been primarily conducted using functional methods with high spatial resolution (e.g., positron emission tomography (PET) or functional MRI (fMRI)). These methods have provided insight into the anatomical and functional changes that occur with age, including changes in brain activity (Cabral et al., 2017; Smitha et al., 2017). These techniques also enable the study of brain connectivity changes. Connectivity measures have high sensitivity for detecting cognitive changes and differences between individuals (e.g., Hedden et al., 2016). Studies showed, for example, that the cognitive decline observed in normal aging may be due to functional connectivity disruptions, particularly in the defaultmode network (DMN; this network is mainly activated when no task is requested from the participant; Andrews-Hanna et al., 2007). The concept of cognitive reserve itself has also emerged in part from fMRI studies, as individuals with a higher cognitive reserve have fewer brain and cognitive alterations than individuals with a lower level of cognitive reserve (Stern, 2009).The contribution of these methods in the precise localization of brain activations and in the study of brain networks is therefore undeniable. However, due to their constrained temporal resolution, age-related changes on the dynamics of the networks involved remain largely understudied. The use of methods with high temporal resolutions, such as magnetoencephalography (MEG) and electroencephalography (EEG) (e.g., Baillet, 2017), can provide sensitive and specific elements on individual differences associated with cognitive aging.

Brain activity is characterized by its spectral complexity, and can be distinguished according to its dominant frequency (delta, theta, alpha, beta, gamma). The delta waves $(1-3 \mathrm{~Hz})$ are the slowest, while the gamma waves $(40+\mathrm{Hz})$ are the fastest. Previous work highlighted that these brain rhythms are associated with different cognitive functions (Buszaki et al., 2006), for example the gamma frequency band is associated with information processing in higher-order cognitive tasks. Previous MEG studies show that networks activated at rest are activated periodically and in different frequency bands (de Pasquale et al., 2010). A decrease in functional connectivity has been observed during aging (Wig, 2017). Moreover, previous work has shown that in older individuals, activations are less frequently observed in the alpha and gamma frequency bands, and more in the delta frequency band (Vlahou, 2014). This slowing of neural activity has been linked to decreased cognitive performance and slower information processing speed. Conversely, the preservation of this neural activity allows cognitive abilities to be maintained with age. However, previous M/EEG studies have mainly focused on the 
average of activations over long periods of time (see Courtney \& Hinault., 2021, for a review), and therefore do not provide insight into the dynamics of brain activities or their association with cognitive changes. It is therefore important to study the fluctuations of brain communications over time.

Spontaneous fluctuations of brain activity have long been considered as noise to be eliminated and/or controlled. They are now considered as a fundamental element of brain communications (e.g., Uddin, 2020). Recent work has demonstrated the importance of sustained synchronization between brain regions for performance in complex cognitive tasks (e.g., Daume et al., 2017). Moreover, disrupted synchronization has been associated with cognitive decline with age (Hinault et al., 2020). However, fluctuations of activity have not been considered in light of individual differences during aging. Impaired stability of brain network dynamics could lead to the neurocognitive changes observed with advancing age (Voytek \& Knight, 2015). The directionality of connectivity between neuronal oscillations may also play a role in the transmission of neuronal communications. Therefore, changes in dynamic connectivity would take place in order to maintain cognitive performance, while failure to make these changes would lead to cognitive decline (Ariza et al., 2015).

Here, we investigated the stability and variability of resting brain networks' synchrony over time in young and elderly healthy participants from the Cam-CAN (Cambridge Centre for Ageing and Neuroscience) database (e.g., Shafto et al., 2014; Taylor et al., 2017). This database includes multimodal neuroimaging data (MEG, f/MRI) as well as cognitive performance assessment in each individual. Analyses were focused on the four networks found activated at rest that are the default network, salience network, left and right fronto-parietal networks. Our objectives were twofold: i) To study changes in dynamic connectivity with age: Between young and old individuals, we hypothesized differences in functional networks, as well as greater variability in the activity of these networks; ii) To investigate the relationships between changes in dynamic connectivity and cognitive changes: We expected that stability in synchronization and directionality of connectivity over time would be associated with better cognitive performance with age, compared to high variability in these measures. Preservation of this neural activity would help maintain cognitive abilities with age.

\section{Methods}

\section{$\underline{\text { Participants }}$}

We analysed data from 46 young (29 women and 17 men; aged 22-29 years) and 46 older healthy adults (29 women and 17 men; aged 60-69 years; see participant demographics characteristic in Table 1). Participants were selected from the Cam-CAN database (e.g., Shafto et al., 2014; Taylor et al., 2017), in line with demographic characteristics of individuals recruited in previous work (e.g., Coquelet et al., 2017; Hinault et al., 2020). All participants were right-handed, showed normal cognitive functioning (Montreal Cognitive Assessment (MoCA) score >26; Nasreddine. et al., 2005), and no neurological or psychiatric condition.

\section{$\underline{\text { Behavioural measures }}$}

A detailed description of the behavioural measures can be found in Shafto et al. (2014) and Taylor et al. (2017). Cognitive performance was assessed with the Mini-Mental State Evaluation (MMSE; Folstein et al., 1975) used as a measure of general cognitive functioning, the Visual Short-Term Memory (VSTM; Vogel et al., 2001) which measures working memory, the Cattell test (Horn \& Cattell, 1966) which is a measure of reasoning ability and the Hotel Test (Shallice \& Burgess, 1991) which assesses planning abilities. 
Resting brain activity was measured for 10 minutes (sampling rate: $1 \mathrm{kHz}$, bandpass filter: 0.03 $330 \mathrm{~Hz}$ ) with a 306-channel MEG system. Participants' 3D-T1 MRI images were acquired on a 32-channel 3T MRI scanner. The following parameters were used: repetition time $=2250 \mathrm{~ms}$; echo time $=2.99 \mathrm{~ms}$; inversion time $=900 \mathrm{~ms}$; flip angle $=9$ degrees; field of view $=256 \mathrm{~mm}$ x $240 \mathrm{~mm}$ x $192 \mathrm{~mm}$; voxel size $=1 \mathrm{~mm}$; GRAPPA acceleration factor $=2$; acquisition time $=$ 4 minutes and 32 seconds.

\section{Data pre-processing}

The Elekta Neuromag MaxFilter 2.2 has been applied to all MEG data (temporal signal space separation (tSSS): 0.98 correlation, 10s window; bad channel correction: ON; motion correction: OFF; 50Hz+harmonics (mains) notch). Afterwards, artifact rejection, filtering (0.3$100 \mathrm{~Hz}$ bandpass), re-referencing (i.e. using the algebraic average of the left and right mastoid electrodes), temporal segmentation into epochs, averaging and source estimation were performed using Brainstorm (Tadel et al., 2011). In addition, physiological artefacts (e.g. blinks, saccades) were identified and removed using spatial space projection of the signal. In order to improve the accuracy of the source reconstruction, the FreeSurfer (Fischl, 2012), software was used to generate cortical surfaces and automatically segment them from the cortical structures from each participant's T1-weighted anatomical MRI. The advanced MEG model was obtained from a symmetric boundary element method (BEM model; OpenMEEG; Gramfort et al., 2010; Kybic et al., 2005), fitted to the spatial positions of each electrode (Huang et al., 1999). A cortically constrained sLORETA procedure was applied to estimate the cortical origin of the scalp MEG signals. The estimated sources were then smoothed and projected into a standard space (i.e., the ICBM152 model) for comparisons between groups and individuals, while accounting for differences in native anatomy. This procedure was applied for the entire recording duration.

\section{$\underline{\text { Network segmentation }}$}

In line with previous work (e.g., Smitha et al., 2017; Van den Heuvel et al., 2009), we investigated the four main brain networks at rest: the default-mode network (DMN), the salience network (SN), the left fronto-parietal network (FPG) and the right fronto-parietal network (FPD). Each network is composed of different brain regions: the DMN is composed of the posterior cingulate cortex, the medial prefrontal and the inferior parietal cortex. The SN is composed of the anterior cingulate cortex, the insula and the pre-complementary motor area. The FPG is composed of the left dorsolateral prefrontal cortex and the left superior parietal cortex. Finally, the FPD is composed of the right dorsolateral prefrontal cortex and the right superior parietal cortex (Figure 1). These networks are involved in different cognitive activities or functions: the DMN is mainly observed at rest and shows lower connectivity levels when participants are currently performing cognitive tasks (Raichle et al., 2001). The SN is associated with the processing of salient stimuli in the environment (Seeley et al., 2007). Finally, the bilateral fronto-parietal network is involved in spatial attention, planning and cognitive control (Kam et al., 2019). We separately investigated the FPG, which is involved in working memory (Murphy et al., 2019) and the FPD which is involved in inhibitory processing (Nee et al., 2007). Regions of interest were selected following segmentation of individual anatomies based on the Desikan-Killiany atlas (Desikan et al., 2006). 

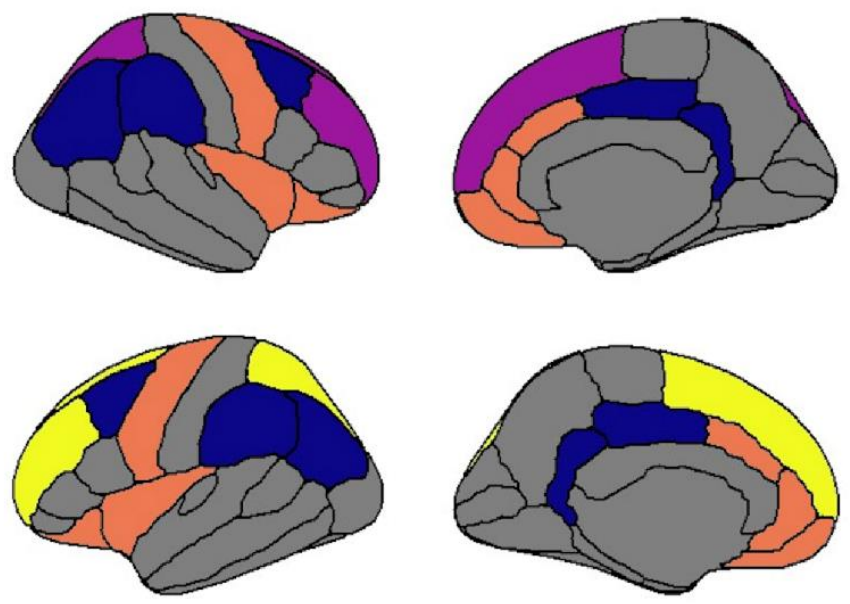

Figure 1: Visualisation of the different regions forming the four studied brain networks; in blue: the DMN; in orange: the SN; in yellow: the FPG; in purple: the FPD

\section{Study of dynamic connectivity}

Phase-locking value analyses (PLV; Lachaux et al., 1999) were used to determine the functional synchrony between regions of interest. PLV estimates the variability of phase differences between two regions over time. If the phase difference varies little, the PLV is close to 1 (this corresponds to high synchronisation between the regions), while the low association of phase difference across regions is indicated by a PLV value close to zero. Because PLV is an undirected measure of functional connectivity, and to investigate brain dynamics with complementary metrics, analyses of transfer entropy (TE) have also been conducted. TE measures of how a signal a can predict subsequent changes in a signal b (Ursino et al., 2020). It then provides a directed measure of a coupling's strength. If there is no coupling between a and $b$, then TE is close to 0 , while TE is close to 1 if there is a strong coupling between a and b.

The range of each frequency band was based on the frequency of the individually observed alpha peak frequency (IAF), measured as the average of peaks detected with both occipitoparietal magnetometers and gradiometers. From previous work (Toppi et al., 2018) the following frequency bands were considered: Delta (IAF-8/IAF-6), Theta (IAF-6/IAF-2), Alpha $(\mathrm{IAF}-2 / \mathrm{IAF}+2), \quad$ Beta $(\mathrm{IAF}+2 / \mathrm{IAF}+14), \quad$ Gamma1 $(\mathrm{IAF}+15 / \mathrm{IAF}+30)$ and Gamma2 $(\mathrm{IAF}+31 / \mathrm{IAF}+80)$. To reduce the dimensionality of the data, the first principal component analysis (PCA) decomposition mode of the time course of activation in each region of interest (ROI) of the Desikan-Killiany atlas brain fragmentation was used. The first component, rather than the average activity, was chosen to reduce signal leakage (Sato et al., 2018). 35 sliding 30s sliding time windows were then extracted for the epochs of interest to calculate the variability across time windows (standard deviation) of the PLV. The analyses were conducted on the average activity within each network, however additional analyses were conducted at the coupling level to further investigate the observed results.

\section{$\underline{\text { Statistical tests }}$}

Functional data (PLV, TE) were analyzed using a 2 (age group: young/old) x 4 (networks: DMN, SN, FPG, and FPD) x 6 (frequency bands: delta, theta, alpha, beta, gamma1, gamma2) 
repeated-measures ANOVA to determine which network and frequency band showed the greatest young/old changes. The Greenhouse-Geisser epsilon correction was used where necessary. Original degrees of freedom and corrected p-values are reported. Significant interactions were followed by permutation t-tests $(N=1,000)$ to compare groups. Finally, correlations as well as regressions aimed at determining the association between these measures and behavioral measures within each group. Results were FDR corrected for multiple comparisons (Benjamini \& Hochberg, 1995).

\section{Results}

\section{Age-related differences in cognitive performance}

The main behavioral and demographic data from the CamCAN database are summarized in Table 1.

\begin{tabular}{|l|l|l|l|}
\hline Variables & Young adults & Older adults & p-value \\
\hline Number of participants & 46 & 46 & - \\
\hline Number of females & 29 & 29 & - \\
\hline Age & 26.5 & 64.5 & - \\
\hline Years of education & $\mathbf{2 2 . 2}$ & $\mathbf{1 9 . 1}$ & $\mathbf{0 . 0 0 1}$ \\
\hline MMSE & $\mathbf{2 9 . 5}$ & $\mathbf{2 8 . 9}$ & $\mathbf{0 . 0 1 3}$ \\
\hline VSTM_all & $\mathbf{0 . 5}$ & $\mathbf{0 . 5}$ & $\mathbf{0 . 0 0 1}$ \\
\hline Cattell & $\mathbf{3 7 . 8}$ & $\mathbf{3 0 . 5}$ & $\mathbf{0 . 0 0 1}$ \\
\hline Hotel_Num_rows & $\mathbf{4 . 7}$ & $\mathbf{4 . 3}$ & $\mathbf{0 . 0 1 8}$ \\
\hline Hotel_Time & $\mathbf{2 2 7 . 7}$ & $\mathbf{3 2 6 . 9}$ & $\mathbf{0 . 0 0 5}$ \\
\hline
\end{tabular}

Table 1: Demographics and scores for both groups younger and older participants

Relative to younger individuals, older adults showed lower scores in the MMSE ( $p=0.013)$, VSTM_all $(\mathrm{p}<0.001)$, Cattell $(\mathrm{p}<0.001)$ and hotel test $(\mathrm{p}=0.018$ for number of rooms; and $\mathrm{p}=0.005$ for time) scores. For the hotel test, a decrease in the rate of correct answers was observed $(\mathrm{p}=0.018)$. A significant increase in response time for the hotel test was also observed in older individuals $(\mathrm{p}=0.005)$.

\section{Increased variability of delta phase synchrony frequency band in older adults}

We first observed a significant effect of network, $F(3,270)=8.085, p<0.001, \eta^{2}=0.082$, frequency, $\mathrm{F}(5,450)=202.748, \mathrm{p}<0.001, \eta^{2}=0.693$, and age, $\mathrm{F}(1,90)=4.698, \mathrm{p}=0.033, \eta 2=$ 0.05 . The interaction between frequency and age, $F(5,450)=6.57, p<0.001, \eta^{2}=0.068$, revealed that this difference in variability between young $(\mathrm{M}=0.076, \mathrm{SE}=0.002)$ and older adults $(\mathrm{M}$ $=0.087, \mathrm{SE}=0.002$ ) was stronger for the delta frequency band. This effect was not observed in other frequency bands. The Age $\mathrm{x}$ Networks interaction for the delta frequency band was also significant, $\mathrm{F}(3,270)=6.823, \mathrm{p}<0.001, \eta^{2}=0.07$, with the DMN network showing the largest difference. These results indicate an increased variability of the delta DMN activity with advancing age (Figure 2). We observed a significant negative correlation between such 
variability and cognitive performance (VSTM, $\mathrm{p}=0.009, \mathrm{r}=-0.387$ ). The rest of the analyses was therefore focused on the DMN network, in the delta frequency band.
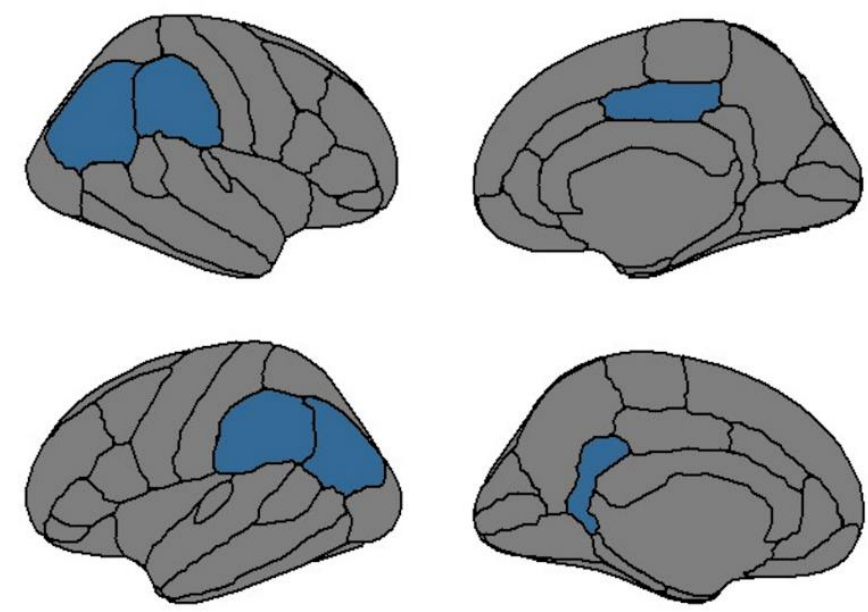

Figure 2: Visualisation of the DMN couplings showing significant differences between young and older adults in the delta frequency band (permutation test, FDR correction; Benjamini \& Hochberg, 1995)

We then performed permutation t-tests on the DMN couplings between age groups. Different couplings were found to be significantly more variable for the older group compared to the younger group especially for interhemispheric and frontoparietal couplings. In the older group, the right frontoparietal coupling was found to be negatively correlated with cognitive performance (MMSE test; $r=-0.305, p=0.039$ ). These data suggest an increase in variability in the overall DMN network in the delta frequency band, but also an increase in the significant variability of specific couplings in this network, both being associated with lower cognitive performance.

\section{Reversal of the direction of information transfer of delta band in older adults}

As phase synchrony measures are undirected, transfer entropy was used to determine whether a specific direction of connectivity was associated with age-related differences. We performed a repeated measures ANOVA Age x Networks x Frequencies x Direction to determine which ,network, which frequency band and in which direction the largest young-to-old changes were found. We showed a significant effect of frequency, $F(5,450)=361.1, p<0.001, \eta^{2}=0.801$. A significant effect of age, $F(1,90)=17.7 p<0.001, \eta^{2}=0.165$ was also observed. Results revealed an increase in the direction of information transfer variability in the delta frequency band, in older adults relative to young adults. An interaction between frequency and age was also observed, $F(5,450)=14.61, p<0.001, \eta^{2}=0.140$. This significant interaction effect indicates larger coupling strength in delta frequency in the older group $(\mathrm{M}=1.218, \mathrm{SE}=0.0231)$ compared to the younger group $(\mathrm{M}=0.921, \mathrm{SE}=0.0231)$.

Student's t-tests were performed to determine the direction of information transfer for young and older adults in the DMN. We saw a significant difference between the fronto-parietal and parieto-frontal direction $(\mathrm{p}=0.013)$, with a significantly larger coupling strength parieto-frontal direction for young relative to older participants. With advancing age, in the DMN network and the delta frequency band, a decrease in the transfer of information from parietal to frontal 
regions has been observed. This decrease in communication can be linked to the cognitive performance observed in this group. Indeed, we conducted regression and correlation analyses to determine the association between these entropy transfer measures and behavioural measures within each group. We found negative correlations of information transfer with cognitive performance in all directions (VSTM, $\mathrm{p}=0.031, \mathrm{r}=-0.319$; Cattell, $\mathrm{p}=0.020, \mathrm{r}=-0.341$ ) in the delta frequency band for older adults.

\section{Discussion}

Our main objective was to investigate changes in the stability and variability of brain communication dynamics with age and the relationship of these changes with age-related cognitive changes. Our connectome-based approach, based on MEG data in healthy young and older participants from the Cam-CAN database, allowed us to investigate changes of connectivity dynamics with aging. Two time-resolved connectivity aspects were studied: the stability of synchronized communications over time, and directed connectivity. Brain activity was studied at rest, as previous work suggested a link between the activity of specific networks at rest and cognitive abilities (e.g. Nashiro et al., 2017). In this study, we first showed an increased variability of phase synchrony over time with age, especially in the delta frequency band. We also showed a reversal of the main direction of synchronized with age: connectivity in the fronto-parietal direction was found to be increased in older participants, whereas it was stronger in the parieto-frontal direction for younger participants. These observations are in line with the available literature on functional connectivity during non-pathological aging (e.g. Geerligs et al., 2015). The results also show for the first time the influence of the stability or variability of functional networks, as well as information exchange over time, on individual cognitive differences during aging. This was made possible by the excellent temporal resolution of MEG, combined with advanced source reconstruction analyses.

The study of oscillatory activity allowed us to specify age-related changes in the variability of phase synchrony over time, and the specific frequency band associated with these differences. Phase synchrony between brain regions is a critical parameter of neural communications (e.g., Fries, 2015). Indeed, with advancing age, changes in synchronized network communications have been observed (see Courtney \& Hinault, 2021, for a review). Our results reveal an increased variability of phase synchrony in the default network, mainly in the delta frequency band with age. Such variability of neural synchrony was negatively correlated with cognitive performance (measures of general cognition, and working memory). This result is consistent with MRI work showing that an age-related decrease in connectivity within the DMN is related to a decrease in memory and executive functions (e.g. Andrews-Hanna et al., 2007). Our results are also consistent with previous $\mathrm{M} / \mathrm{EEG}$ work reporting an overall slowing of brain activity with advancing age (e.g., Celesia, 1986), with an increase of slow rhythms relative to faster rhythms. Increased slow waves seem to be associated with the cognitive decline observed with advancing age. Here, we show that this slowing of brain rhythms with age is associated with a loss of stability in neuronal communications, and poorer performance.

In association with synchrony analyses, transfer entropy analyses allow the quantification of directed connectivity (see Ursino et al., 2020). This enables to quantify the information flow between brain regions more precisely than functional connectivity, thus allowing the detection of causal interactions (i.e., $A$ must precede $B$ ) between brain regions. Such investigation of directed connectivity revealed a decrease in the parietofrontal direction of brain communications relative to the frontoparietal direction in the default network and the delta frequency band with age. This reversal of information transfer between young and old participants was negatively correlated with cognitive performance (especially for working memory and fluid intelligence). The reversal of information transfer and decreased variability 
in phase synchrony observed here may help furthering the age-related pattern described in the PASA model (Cabeza et al., 2018). According to this model, the increase in the recruitment of frontal regions in elderly people would be an indicator of their attempts to compensate for the decrease in their cognitive abilities. Here, we show a decrease in information transfer to these frontal regions (therefore, a decrease in recruitment from these regions), which is negatively associated with cognitive performances. These results allow us to understand this concept at the network communication dynamics levels. Further investigation of investigation transfer during task completion will be necessary to specify its associations with the direct implementation of cognitive processes.

Several methodological considerations must be discussed regarding the reported results. First, the investigation of resting-state activity prevents in part the direct investigation of the neural bases of cognitive processes, which may explain the small number of associations with cognition. This could also reflect the fact that the Cam-CAN database does not include tasks directly testing executive functions. However, studying dynamic network connectivity at rest furthers our knowledge on the stability of these networks and help better characterize their individual variations.

Theoretically, the variability of brain communications has received little investigation, as it was long considered as noise, but is now recognized as contributing to brain functions (Uddin et al., 2020). Here, we show that healthy aging is associated with an increased variability in synchronized brain communications, and with changes of the main connectivity directions between brain regions. Results highlight that even when brain networks are not engaged in a particular cognitive activity, significant changes occur with age regarding connectivity dynamics and information flow between regions of different functional brain networks. Advancing age appears to be accompanied by a functional disorganization of dynamic networks, with a loss of communication stability and a decrease in the information transmitted. The study of dynamic connectivity contributes to a better understanding of the cognitive decline with aging. The stability of communications and its alteration should be considered in the framework of maintenance, reserve and resilience (Cabeza 2018; Stern, 2020). 
bioRxiv preprint doi: https://doi.org/10.1101/2022.01.26.477817; this version posted January 28, 2022. The copyright holder for this preprint (which was not certified by peer review) is the author/funder, who has granted bioRxiv a license to display the preprint in perpetuity. It is made available under aCC-BY 4.0 International license.

Funding: This research did not receive any specific grant from funding agencies in the public, commercial, or not-for-profit sectors. 


\section{References}

Andrews-Hanna, J. R., Snyder, A. Z., Vincent, J. L., Lustig, C., Head, D., Raichle, M. E., \& Buckner, R. L. Disruption of large-scale brain systems in advanced aging. Neuron, 56(5) (2007), 924-935. doi: 10.1016/J.NEURON.2007.10.038

Ariza, P., Solesio-Jofre, E., Martínez, J. H., Pineda-Pardo, J. A., Niso, G., Maestú, F., \& Buldú, J. M. Evaluating the effect of aging on interference resolution with time-varying complex networks analysis. Frontiers in Human Neuroscience, (2015). doi: 10.3389/fnhum.2015.00255

Baillet, S. Magnetoencephalography for brain electrophysiology and imaging. Nature Neuroscience, 20(3) (2017), 327-339. doi: 10.1038/nn.4504

Benjamini, Y., \& Hochberg, Y. Controlling the false discovery rate: a practical and powerful approach to multiple testing. Journal of the Royal statistical society: series $B$ (Methodological), 57(1) (1995), 289-300. doi: 193.54.110.55

Buzsaki, G. Rhythms of the Brain. Oxford university press. (2006). doi: 10.1093/acprof:oso/9780195301069.001.0001

Cabeza, R., Albert, M., Belleville, S., Craik, F. I. M., Duarte, A., Grady, C. L., Lindenberger, U., Nyberg, L., Park, D. C., Reuter-Lorenz, P. A., Rugg, M. D.,Steffener, J., \& Rajah, M. N. Maintenance, reserve and compensation: The cognitive neuroscience of healthy ageing. Nature Reviews Neuroscience. 19(11) (2018), 701. doi: 10.1038/s41583018-0068-2

Cabral, J., Kringelbach, M. L., \& Deco, G. Functional connectivity dynamically evolves on multiple time-scales over a static structural connectome: Models and mechanisms. NeuroImage, 160 (2017), 84-96. doi: 10.1016/j.neuroimage.2017.03.045

Celesia, G. G. EEG and event-related potentials in aging and dementia. Journal of Clinical Neurophysiology, 3(2) (1986), 99-111.

Coquelet, N., Mary, A., Peigneux, P., Goldman, S., Wens, V., \& De Tiège, X. The electrophysiological connectome is maintained in healthy elders: A power envelope correlation MEG study. Scientific Reports, 7(1) (2017), 1-10. doi: 10.1038/s41598-017$13829-8$

Courtney, S. M., \& Hinault, T. When the time is right: Temporal dynamics of brain activity in healthy aging and dementia. Progress in neurobiology, 203 (2021), 102076. doi: 10.1016/J.PNEUROBIO.2021.102076

Daume, J., Gruber, T., Engel, A. K., \& Friese, U. Phase-Amplitude Coupling and Long-Range Phase Synchronization Reveal Frontotemporal Interactions during Visual Working Memory. The Journal of Neuroscience, 37(2) (2017), 313 LP - 322. doi: 10.1523/JNEUROSCI.2130-16.2016

Daselaar, S. M., Iyengar, V., Davis, S. W., Eklund, K., Hayes, S. M., \& Cabeza, R. E. Less wiring, more firing: low-performing older adults compensate for impaired white matter 
with greater neural activity. Cerebral cortex, 25(4) (2015), 983-990. doi: 10.1093/CERCOR/BHT289

Desikan, R. S., Ségonne, F., Fischl, B., Quinn, B. T., Dickerson, B. C., Blacker, D., Buckner R.L., Dale A.M., Maguire R.P., Hyman B.T., Albert M.S., Killiany, R. J. An automated labeling system for subdividing the human cerebral cortex on MRI scans into gyral based regions of interest. Neuroimage, 31(3) (2006), 968-980. doi : 10.1016/J.NEUROIMAGE.2006.01.021

Fischl, B. FreeSurfer. Neuroimage, 62(2) (2012), 774-781. doi : 10.1016/j.neuroimage.2012.01.021

Folstein, M. F., Folstein, S. E., \& McHugh, P. R. "Mini-mental state": a practical method for grading the cognitive state of patients for the clinician. Journal of psychiatric research, 12(3) (1975), 189-198. doi: 10.1016/0022-3956(75)90026-6

Fries P. Rhythms for Cognition: Communication through Coherence. Neuron. (2015);88(1):220-235. doi:10.1016/j.neuron.2015.09.034

Geerligs, L., Renken, R. J., Saliasi, E., Maurits, N. M., \& Lorist, M. M. A brain-wide study of age-related changes in functional connectivity. Cerebral cortex, 25(7) (2015), 1987-1999. doi: 10.1093/cercor/bhu012

Gramfort, A., Papadopoulo, T., Olivi, E., \& Clerc, M. OpenMEEG: opensource software for quasistatic bioelectromagnetics. Biomedical engineering online, 9(1) (2010), 1-20. doi: $10.1186 / 1475-925 X-9-45$

Hedden, T., \& Gabrieli, J. D. E. Insights into the ageing mind: A view from cognitive neuroscience. In Nature Reviews Neuroscience (Vol. 5, Issue 2, pp. 87-96) (2004). European Association for Cardio-Thoracic Surgery. doi: 10.1038/nrn1323

Hedden, T., Schultz, A. P., Rieckmann, A., Mormino, E. C., Johnson, K. A., Sperling, R. A., \& Buckner, R. L. Multiple Brain Markers are Linked to Age-Related Variation in Cognition. Cerebral Cortex (New York, NY), 26(4) (2016), 1388. doi: 10.1093/CERCOR/BHU238

Hinault, T, Kraut, M., Bakker, A., Dagher, A., \& Courtney, S. Disrupted neural synchrony mediates the relationship between white matter integrity and cognitive performance in older adults. Cerebral Cortex (2020), bhaa141. doi: 10.1093/cercor/bhaa141

Horn, J. L., \& Cattell, R. B. Refinement and test of the theory of fluid and crystallized general intelligences. Journal of educational psychology, 57(5) (1966), 253. doi: $10.1037 / \mathrm{h} 0023816$

Huang, M. X., John C. Mosher, and R. M. Leahy. "A sensor-weighted overlapping-sphere head model and exhaustive head model comparison for MEG." Physics in Medicine \& Biology 44.2 (1999): 423. doi: 10.1088/0031-9155/44/2/010

Hultsch, D. F., Strauss, E., Hunter, M. A., \& MacDonald, S. W. S. Intraindividual variability, cognition, and aging. In The handbook of aging and cognition, 3rd ed(pp. 491-556). Psychology Press (2008) 
bioRxiv preprint doi: https://doi.org/10.1101/2022.01.26.477817; this version posted January 28, 2022. The copyright holder for this preprint (which was not certified by peer review) is the author/funder, who has granted bioRxiv a license to display the preprint in perpetuity. It is made available under aCC-BY 4.0 International license.

Kam, J. W. Y., Lin, J. J., Solbakk, A. K., Endestad, T., Larsson, P. G., \& Knight, R. T. Default network and frontoparietal control network theta connectivity supports internal attention. Nature Human Behaviour, 3(12) (2019), 1263-1270. doi: 10.1038/S41562-019-0717-0

Kybic, J., Clerc, M., Abboud, T., Faugeras, O., Keriven, R., \& Papadopoulo, T. A common formalism for the integral formulations of the forward EEG problem. IEEE transactions on medical imaging, 24(1) (2005), 12-28. doi: 10.1109/TMI.2004.837363

Lachaux, J. P., Rodriguez, E., Martinerie, J., \& Varela, F. J. Measuring phase synchrony in brain signals. Human brain mapping, 8(4) (1999), 194-208. doi: 10.1002/(SICI)10970193(1999)8:4

Murphy, A. C., Bertolero, M. A., Papadopoulos, L., Lydon-Staley, D. M., \& Bassett, D. S. Multimodal network dynamics underpinning working memory. Nature Communications, 11(1) (2020). https://doi.org/10.1038/S41467-020-15541-0

Nashiro, K., Sakaki, M., Braskie, M. N., \& Mather, M. Resting-state networks associated with cognitive processing show more age-related decline than those associated with emotional processing. Neurobiology of aging, 54 (2017), 152-162. doi: 10.1016/j.neurobiolaging.2017.03.003

Nasreddine, Z. S., Phillips, N. A., Bédirian, V., Charbonneau, S., Whitehead, V., Collin, I., Cummings, J.L., Chertkow, H. The Montreal Cognitive Assessment, MoCA: a brief screening tool for mild cognitive impairment. Journal of the American Geriatrics Society, 53(4) (2005), 695-699. doi: 10.1111/j.1532-5415.2005.53221.x

Nee, D. E., Wager, T. D., \& Jonides, J. Interference resolution: Insights from a meta-analysis of neuroimaging tasks. Cognitive, Affective, \& Behavioral Neuroscience 2007 7:1, 7(1), 1-17.doi : 10.3758/CABN.7.1.1

de Pasquale, F., Penna, S. Della, Snyder, A. Z., Lewis, C., Mantini, D., Marzetti, L., Belardinelli, P., Ciancetta, L., Pizzella, V., Romani, G. L., \& Corbetta, M. Temporal dynamics of spontaneous MEG activity in brain networks. Proceedings of the National Academy of Sciences, 107(13) (2010), 6040-6045. doi: 10.1073/PNAS.0913863107

Raichle, M. E., MacLeod, A. M., Snyder, A. Z., Powers, W. J., Gusnard, D. A., \& Shulman, G. L. A default mode of brain function. Proceedings of the National Academy of Sciences, 98(2) (2001), 676-682. doi: 10.1073/PNAS.98.2.676

Sadaghiani, S., \& Wirsich, J. Intrinsic connectome organization across temporal scales: New insights from cross-modal approaches. Network Neuroscience, 4(1) (2020), 1-29. doi: 10.1162/netn_a_00114

Sato, M., Yamashita, O., Sato, M. A., \& Miyawaki, Y. Information spreading by a combination of MEG source estimation and multivariate pattern classification. PloS one, 13(6) (2018), e0198806. doi: 10.1371/journal.pone.0198806

Seeley, W. W., Menon, V., Schatzberg, A. F., Keller, J., Glover, G. H., Kenna, H., Reiss, A. L., \& Greicius, M. D. Dissociable Intrinsic Connectivity Networks for Salience Processing 
bioRxiv preprint doi: https://doi.org/10.1101/2022.01.26.477817; this version posted January 28, 2022. The copyright holder for this preprint (which was not certified by peer review) is the author/funder, who has granted bioRxiv a license to display the preprint in perpetuity. It is made available under aCC-BY 4.0 International license.

and Executive Control. The Journal of Neuroscience, 27(9) (2007), 2349. doi: 10.1523/JNEUROSCI.5587-06.2007

Shafto, M. A., Tyler, L. K., Dixon, M., Taylor, J. R., Rowe, J. B., Cusack, R., Calder, A. J., Marslen-Wilson, W. D., Duncan, J., \& Dalgleish, T. The Cambridge Centre for Ageing and Neuroscience (Cam-CAN) study protocol: A cross-sectional, lifespan, multidisciplinary examination of healthy cognitive ageing. BMC Neurology, 14(1) (2014), 204. doi: 10.1186/S12883-014-0204-1

Shallice, T. I. M., \& Burgess, P. W. Deficits in strategy application following frontal lobe damage in man. Brain, 114(2) (1991), 727-741. doi: 10.1093/brain/114.2.727

Smitha, K. A., Akhil Raja, K., Arun, K. M., Rajesh, P. G., Thomas, B., Kapilamoorthy, T. R., $\&$ Kesavadas, C. Resting state fMRI: A review on methods in resting state connectivity analysis and resting state networks. The neuroradiology journal, 30(4) (2017), 305-317. doi: $10.1177 / 1971400917697342$

Stern, Y. Cognitive reserve. Neuropsychologia, 47(10) (2009), 2015-2028. doi: 10.1016/J.NEUROPSYCHOLOGIA.2009.03.004

Stern, Y., Arenaza-Urquijo, E. M., Bartrés-Faz, D., Belleville, S., Cantilon, M., Chetelat, G., Ewers, M., Franzmeier, N., Kempermann, G., Kremen, W. S., Okonkwo, O., Scarmeas, N., Soldan, A., Udeh-Momoh, C., Valenzuela, M., Vemuri, P., \& Vuoksimaa, E. Whitepaper: Defining and investigating cognitive reserve, brain reserve, and brain maintenance. Alzheimer's \& Dementia, 16(9) (2020), 1305-1311. doi: 10.1016/j.jalz.2018.07.219

Tadel, F., Baillet, S., Mosher, J. C., Pantazis, D., \& Leahy, R. M. Brainstorm: a user-friendly application for MEG/EEG analysis. Computational intelligence and neuroscience, (2011). doi : $10.1155 / 2011 / 879716$

Taylor, J. R., Williams, N., Cusack, R., Auer, T., Shafto, M. A., Dixon, M., Tyler, L. K., CamCAN, \& Henson, R. N. The Cambridge Centre for Ageing and Neuroscience (Cam-CAN) data repository: Structural and functional MRI, MEG, and cognitive data from a crosssectional adult lifespan sample. NeuroImage, 144 (2017), 262-269. doi: 10.1016/J.NEUROIMAGE.2015.09.018

Uddin, L. Q. Bring the Noise: Reconceptualizing Spontaneous Neural Activity. Trends in Cognitive Sciences, 24(9) (2020), 734-746. doi: 10.1016/J.TICS.2020.06.003

Ursino, M., Ricci, G., \& Magosso, E. Transfer Entropy as a Measure of Brain Connectivity: A Critical Analysis With the Help of Neural Mass Models. Frontiers in Computational Neuroscience (2020), 14, 45. Doi: 10.3389/FNCOM.2020.00045/BIBTEX

Van Den Heuvel, M. P., Mandl, R. C., Kahn, R. S., \& Hulshoff Pol, H. E. Functionally linked resting-state networks reflect the underlying structural connectivity architecture of the human brain. Human brain mapping, 30(10) (2009), 3127-3141. doi: 10.1002/hbm.20737 
Vlahou, E. L., Thurm, F., Kolassa, I. T., \& Schlee, W. Resting-state slow wave power, healthy aging and cognitive performance. Scientific reports (2014), 4, 5101. doi: 10.1038/srep05101

Vogel, E. K., Woodman, G. F., \& Luck, S. J. Storage of features, conjunctions, and objects in visual working memory. Journal of experimental psychology: human perception and performance, 27(1) (2001), 92. doi: 10.1037//0096-1523.27.1.92

Voytek B., \& Knight RT. Dynamic network communication as a unifying neural basis for cognition, development, aging, and disease. Biological Psychiatry, 77(12) (2015), 10891097. doi: 10.1016/J.BIOPSYCH.2015.04.016

Wig, G. S. Segregated systems of human brain networks. Trends in Cognitive Sciences, 21(12) (2017), 981-996. doi: 10.1016/j.tics.2017.09.006 